\title{
Immunogenicity Specimen Assessments Supplemental Qualifiers Dataset
}

National Cancer Institute

\section{Source}

National Cancer Institute. Immunogenicity Specimen Assessments Supplemental

Qualifiers Dataset. NCI Thesaurus. Code C147219.

A dataset containing supplemental information, specifically non-standard variables, to parent records in the immunogenicity specimen assessments domain. 